\title{
Erratum to: Anti-angiogenic therapy for cancer: current progress, unresolved questions and future directions
}

\author{
Naveen S. Vasudev • Andrew R. Reynolds
}

Published online: 4 June 2014

(C) Springer Science+Business Media Dordrecht 2014

\section{Erratum to: Angiogenesis}

DOI 10.1007/s10456-014-9420-y

The author wishes to correct the errors in Table 1 of the original publication.

The content in Table 1, under the section entitled 'Colorectal cancer' has three errors.

1. For the AVF2107 trial, in the treatment column, "FOLFIRI" should be replaced with "IFL"
2. For the NO16966 trial, in the outcome column "Improvement in OS and PFS" should be replaced with "Improvement in PFS" and

3. Definitions of the abbreviations IFL and XELOX were omitted and have been added to the foot of the table.

The correct Table 1 is provided in this Erratum.

The online version of the original article can be found under doi:10.1007/s10456-014-9420-y.

N. S. Vasudev · A. R. Reynolds ( $\square)$

Tumour Biology Team, Breakthrough Breast Cancer Research

Centre, The Institute of Cancer Research, Fulham Road,

London SW3 6JB, UK

e-mail: andrew.reynolds@icr.ac.uk

Present Address:

N. S. Vasudev

Cancer Research UK Centre, Leeds Institute of Cancer and

Pathology, St James's University Hospital, Beckett Street,

Leeds LS9 7TF, UK 
Table 1 Randomised trials of anti-angiogenic agents cited in this article

\begin{tabular}{|c|c|c|c|}
\hline Indication & Treatment & $\begin{array}{l}\text { Trial identifier } \\
\text { and citation }\end{array}$ & Outcome \\
\hline \multicolumn{4}{|l|}{ Breast cancer } \\
\hline \multirow[t]{6}{*}{ Metastatic 1st line } & Paclitaxel \pm bevacizumab & E2100 [40] & Improvement in PFS not OS \\
\hline & Docetaxel \pm bevacizumab (HER-2 negative population) & AVADO [41] & Improvement in PFS, OS NA \\
\hline & $\begin{array}{l}\text { Capecitabine, taxane or anthracycline } \pm \text { bevacizumab } \\
\text { (HER-2 negative population) }\end{array}$ & RIBBON-1 [42] & Improvement in PFS but not in OS \\
\hline & $\begin{array}{l}\text { Docetaxel and trastuzumab } \pm \text { bevacizumab (HER-2 } \\
\text { positive population) }\end{array}$ & AVEREL [104] & No improvement in PFS, OS NA \\
\hline & Docetaxel \pm sunitinib (HER-2 negative population) & Sun 1064 [45] & No improvement in PFS or OS \\
\hline & $\begin{array}{l}\text { Paclitaxel } \pm \text { bevacizumab or sunitinib (HER-2 negative } \\
\text { population) }\end{array}$ & SUN 1094 [46] & Inferior PFS for sunitinib arm \\
\hline \multirow{4}{*}{$\begin{array}{l}\text { Metastatic 2nd line } \\
\text { and beyond }\end{array}$} & Capecitabine \pm bevacizumab & AVF2119 [39] & No improvement in PFS or OS \\
\hline & $\begin{array}{l}\text { Capecitabine, taxane, gemcitabine, or } \\
\text { vinorelbine } \pm \text { bevacizumab (HER-2 negative } \\
\text { population) }\end{array}$ & RIBBON-2 [43] & Improvement in PFS but not in OS \\
\hline & Capecitabine \pm sunitinib & $\begin{array}{l}\text { NCT00435409 } \\
\text { [44] }\end{array}$ & No improvement in PFS or OS \\
\hline & Capecitabine vs. sunitinib (HER-2 negative population) & SUN 1107 [47] & Inferior PFS and OS for sunitinib arm \\
\hline Adjuvant & $\begin{array}{l}\text { Anthracycline, taxane or both } \pm \text { bevacizumab (triple } \\
\text { negative population) }\end{array}$ & $\begin{array}{l}\text { BEATRICE } \\
{[58]}\end{array}$ & No improvement in DFS, OS NA \\
\hline \multirow[t]{2}{*}{ Neo-adjuvant } & $\begin{array}{l}\text { Doxorubicin/docetaxel/ } \\
\text { cyclophosphamide } \pm \text { bevacizumab }\end{array}$ & $\begin{array}{l}\text { NCT00408408 } \\
\text { [63] }\end{array}$ & $\begin{array}{l}\text { Improvement in pathological complete } \\
\text { response rate (primary } \\
\text { endpoint) }\end{array}$ \\
\hline & $\begin{array}{l}\text { Epirubicin/docetaxel/Cyclophosphamide } \pm \text { bevacizumab } \\
\text { (HER-2 negative population) }\end{array}$ & $\begin{array}{l}\text { NCT00567554 } \\
{[64]}\end{array}$ & $\begin{array}{l}\text { Improvement in pathological complete } \\
\text { response rate (primary endpoint) }\end{array}$ \\
\hline \multicolumn{4}{|l|}{ Colorectal cancer } \\
\hline \multirow[t]{5}{*}{ Metastatic 1st line } & IFL \pm bevacizumab & AVF2107 [19] & Improvement in OS and PFS \\
\hline & FOLFOX or XELOX \pm bevacizumab & NO16966 [21] & Improvement in PFS \\
\hline & Capecitabine \pm bevacizumab & AVEX [22] & Improvement in PFS, OS NA \\
\hline & FOLFIRI \pm sunitinib & SUN1122 [28] & No improvement in PFS \\
\hline & FOLFOX \pm vatalanib & $\begin{array}{l}\text { CONFIRM } 1 \\
\text { [29] }\end{array}$ & No improvement in PFS or OS \\
\hline \multirow{4}{*}{$\begin{array}{l}\text { Metastatic 2nd line } \\
\text { and beyond }\end{array}$} & FOLFOX \pm bevacizumab & E3200 [20] & Improvement in OS and PFS \\
\hline & FOLFOX \pm vatalanib & $\begin{array}{l}\text { CONFIRM } 2 \\
\text { [30] }\end{array}$ & Improvement in PFS but not OS \\
\hline & FOLFIRI \pm aflibercept & VELOUR [27] & Improvement in OS and PFS \\
\hline & Regorafenib versus placebo & CORRECT [31] & Improvement in OS \\
\hline $\begin{array}{l}\text { Continuation } \\
\text { beyond } \\
\text { progression }\end{array}$ & Chemotherapy \pm bevacizumab & ML18 147 [92] & Improvement in OS \\
\hline \multirow[t]{2}{*}{ Adjuvant } & FOLFOX \pm bevacizumab & $\begin{array}{l}\text { NSABP C-08 } \\
{[56]}\end{array}$ & No improvement in OS \\
\hline & FOLFOX or XELOX \pm bevacizumab & AVANT [57] & No improvement in OS \\
\hline \multicolumn{4}{|c|}{ Hepatocellular carcinoma } \\
\hline \multirow[t]{2}{*}{ Metastatic 1st line } & Sorafenib versus placebo & $\begin{array}{l}\text { NCT00105443 } \\
\text { [17] }\end{array}$ & Improvement in PFS and OS \\
\hline & Brivanib versus sorafenib & $\begin{array}{l}\text { BRISK-FL } \\
\text { [145] }\end{array}$ & $\begin{array}{l}\text { OS non-inferiority end-point for brivanib } \\
\text { versus sorafenib not met }\end{array}$ \\
\hline Metastatic 2nd line & Brivanib versus placebo & $\begin{array}{l}\text { BRISK-PS } \\
\text { [146] }\end{array}$ & Improvement in PFS but not OS \\
\hline
\end{tabular}


Table 1 continued

\begin{tabular}{|c|c|c|c|}
\hline Indication & Treatment & $\begin{array}{l}\text { Trial identifier } \\
\text { and citation }\end{array}$ & Outcome \\
\hline \multicolumn{4}{|l|}{ Melanoma } \\
\hline \multirow[t]{2}{*}{ Metastatic 1st line } & Paclitaxel/carboplatin \pm bevacizumab & $\mathrm{BEAM}^{* * *}[48]$ & No improvement in PFS or OS \\
\hline & Paclitaxel/carboplatin \pm sorafenib & NCT00110019 [49] & No improvement in PFS or OS \\
\hline Metastatic 2nd line & Paclitaxel/carboplatin \pm sorafenib & NCT00111007 [50] & No improvement in PFS or OS \\
\hline \multicolumn{4}{|l|}{$N S C L C^{*}$} \\
\hline \multirow[t]{2}{*}{ Metastatic 1st line } & Paclitaxel/carboplatin \pm bevacizumab & NCT00021060 [32] & Improvement in PFS and OS \\
\hline & Cisplatin/gemcitabine \pm bevacizumab & AVAiL [33] & Improvement in PFS but not OS \\
\hline \multicolumn{4}{|l|}{ Ovarian cancer } \\
\hline \multirow[t]{2}{*}{ Metastatic 1st line } & Paclitaxel/carboplatin \pm bevacizumab & ICON-7 [36] & Improvement in PFS, OS NA \\
\hline & Paclitaxel/carboplatin \pm bevacizumab & GOG218 [37] & $\begin{array}{l}\text { Improvement in PFS, OS confounded } \\
\text { by cross-over }\end{array}$ \\
\hline Metastatic 2nd line & Gemcitabine/carboplatin \pm bevacizumab & OCEANS [38] & Improvement in PFS but not OS \\
\hline \multicolumn{4}{|l|}{ Pancreatic cancer } \\
\hline Metastatic 1st line & Gemcitabine \pm bevacizumab & CALGB 80303 [51] & No improvement in PFS or OS \\
\hline \multicolumn{4}{|l|}{ PNET } \\
\hline Metastatic 1st line & Sunitinib versus placebo & NCT00428597 [18] & Improvement in PFS, OS NA \\
\hline \multicolumn{4}{|l|}{ Prostate cancer** } \\
\hline \multirow[t]{2}{*}{ Metastatic 1st line } & Docetaxel/prednisone \pm bevacizumab & CALGB 90401 [52] & Improvement in PFS but not OS \\
\hline & Docetaxel/prednisone \pm aflibercept & VENICE [53] & No improvement in PFS or OS \\
\hline \multicolumn{4}{|l|}{ Renal cancer } \\
\hline \multirow[t]{4}{*}{ Metastatic 1st line } & Sorafenib versus placebo & TARGET [9] & Improvement in PFS and OS \\
\hline & Sunitinib versus interferon-alpha & NCT00098657 [11] & Improvement in PFS and OS \\
\hline & Pazopanib versus placebo & NCT00334282 [13] & $\begin{array}{l}\text { Improvement in PFS, OS confounded } \\
\text { by cross-over }\end{array}$ \\
\hline & Sunitinib versus pazopanib & COMPARZ [15] & PFS and OS were similar \\
\hline Metastatic 2nd line & Axitinib versus sorafenib & AXIS [16] & $\begin{array}{l}\text { Improvement in favour of axitinib } \\
\text { for PFS but not OS }\end{array}$ \\
\hline
\end{tabular}

DFS disease-free survival, FOLFIRI 5-FU, leucovorin and irinotecan, FOLFOX 5-FU, leucovorin and oxaliplatin, HER-2 human epidermal growth factor receptor-2, IFL irinotecan, 5FU and leucovorin, $N A$ not available (pending, unknown or not reported), NSCLC non-small cell lung cancer, $O S$ overall survival, PNET pancreatic neuroendocrine tumour, PFS progression-free survival, XELOX capecitabine and oxaliplatin

* Non-squamous NSCLC only; ** castration resistant; *** randomised phase II study 